\title{
Development and Flow Modes of Vertical Taylor-Couette System with Free Surface
}

\author{
Takashi Watanabe ${ }^{1 *}$, Yorinobu Toya ${ }^{2}$, Shota Hara ${ }^{1}$ \\ ${ }^{1}$ Graduate School of Information Science, Nagoya University, Nagoya, Japan \\ ${ }^{2}$ Department of Mechanical Engineering, Nagano National College of Technology, Nagano, Japan \\ Email: ${ }^{\text {info@vi.cs.is.nagoya-u.ac.jp }}$
}

Received 24 January 2014; revised 21 February 2014; accepted 11 March 2014

Copyright (C) 2014 by authors and Scientific Research Publishing Inc.

This work is licensed under the Creative Commons Attribution International License (CC BY).

http://creativecommons.org/licenses/by/4.0/

(c) () Open Access

\begin{abstract}
We have numerically and experimentally investigated the flow modes of Taylor-Couette system consisting of coaxial two cylinders with vertical axes. The inner cylinder rotates and the outer cylinder and the bottom end of the cylinders remain stationary. The upper top boundary is the free surface of the working liquid between the inner and outer cylinders and it contacts with the air. While this flow appears in fluid machinery and chemical reactors and includes industrial interests, it also contains problems of fluid mechanics, which is about the behavior of the free surface in the rotating field. In this paper, we concretely show the developments of the one cell mode flow and the three cell mode flow at a small aspect ratio. We also represent the bifurcation diagram of the flow at the moderate aspect ratio about 5.5. In the numerical simulation, the flow is rest in the initial state, and the inner cylinder is linearly or suddenly accelerated to attain a flow with a prescribed Reynolds number. When the acceleration of the inner cylinder is high, an imperfect bifurcation occurs and the flows of the secondary modes emerge. At high Reynolds numbers, the flow first has many vortices and then some of the vortices collapse and the final stable flow arises. The loci of the normal five cell mode, the anomalous six cell mode and the secondary seven cell mode are determined.
\end{abstract}

\section{Keywords}

Taylor-Couette System; Vertical Cylinders; Free Surface; Flow Mode; Mode Transition; Bifurcation

\section{Introduction}

Taylor-Couette system has been investigated as a typical model of bioreactors, filtration devices and fluid machinery. In addition, this system presents a basic problem about rotating flows and its dynamical characteristics

${ }^{*}$ Corresponding author. 
have been examined. In this paper, the mode transition of the flow between two coaxial cylinders with vertical axes is investigated. The inner cylinder rotates and the outer cylinder is stationary. The bottom end wall is fixed and the glycerin solution is filled in the annulus between the two cylinders. At the top, the solution contacts with the air and the surface is free.

One of the reasons why the Taylor-Couette system attracts many interests is the finiteness of the cylinder lengths and the bifurcation of the flow phenomena. While an analytical approach can be used for the flow between two cylinders with infinite lengths or periodical conditions, the finite lengths of the cylinders introduce an additional effect of the end wall(s) and the straightforward approach cannot be applied. This additional effect makes the flow bifurcation imperfect, and the flow patterns different from those found in the perfect condition appear [1] [2].

The studies of the flow which develops between two vertical cylinders and has a free surface at the top are classified in three groups. The first group assumed the infinite length of cylinders even though they are finite. The second group examined the stratified flow, and the third group investigated an asymmetric system with the free surface at the top and the stationary end wall at the bottom.

In the first group, Cole [3] measured the torque on the cylinder and estimated the condition of the transition from the axisymmetric flow to the wavy flow. The inner cylinder was submerged in the working fluid. The finiteness of the cylinder lengths was assumed and no attention was paid on the flow condition near the end walls of the inner cylinder. For the flow with the submerged cylinder, Ammar et al. [4] measured the axial velocity component and Deng et al. [5] investigated the profiles of the free surface and velocity.

The stratified flow was regarded as a model of the flows in atmosphere and ocean. Caton et al. [6] used the linear stability theory and studied the rotating flow where the density linearly changed in the vertical direction. Ermanyuk et al. [7] experimentally investigated a salt-stratified two-layer fluid and found the flows in which waves appeared only at the interfaces between Taylor vortices and the flows with waves both at the interfaces and inside of the vortices. Similar wavy flow was also reported by Woods et al. [8]. Oglethorpe et al. [9] investigated the linearly stratified vertical Taylor-Couette system and found Richardson number dependent phenomena where the density was almost constant in each Taylor vortex and density jump appeared between vortices.

When the height of the working fluid is lower than that of the cylinders, the free surface appears between cylinder walls. The finite flow field with the stationary bottom end wall and the top free surface is called an asymmetric system in the vertical direction. When the convection effect is large, the entrainment of the air into the working fluid occurs at the free surface. Djéridi et al. [10] [11] classified the bubbly Taylor-Couettte flows into a dispersed flow, ring flow and a spiral flow. Atkhen et al. [12] investigated the effects of the rotation and the superimposed axial flow on the dispersion of the bubbles, and Sugiyama et al. [13] simulated the motions of bubbles. These studies found the drag and torque reduction in the bubbly flows.

On the other hand, the characteristics of the asymmetric flow without the entrainment of the air have also been investigated. Similarly in the symmetric system with both stationary end walls [14], Nakamura et al. [15] and Toya et al. [16] found normal, secondary and anomalous flow mods in the asymmetric system and carried out a systematic research to investigate the transition processes during the quasi-steady decrease of the Reynolds number. Linek et al. [17] studied the developing processes of the asymmetric system. Following these studies, Watanabe et al. [18] numerically investigated the asymmetric system at very small aspect ratio (ratio of the cylinder length to the gap width between two cylinders) below 4.2, and found a reasonable coincidence with the experimental results.

In this paper, we first show the flow development processes at small aspect ratio. Then, we numerically confirm the experimental results [15] [16] and reveal the bifurcation phenomena of the dynamics in the asymmetric system at the aspect ratio from 5.0 to 6.0 .

\section{Formulation}

\subsection{Flow Configuration}

Taylor-Couette system consists of the rotating inner cylinder and the stationary outer cylinder. The cylinders are coaxial and their axes are parallel to the direction of the gravitational force. The radii of the inner and outer cylinders are $r_{\text {in }}$ and $r_{\text {out }}$, respectively. The gap width $d$ of the cylinders is given by $r_{\text {out }}-r_{\text {in }}$. The bottom end of the cylinders is a stationary wall and no wall appears at the top. The height of the working fluid is $L$ and it is lower than the height of the cylinders. This means that the free surface appears between the inner and outer cylinders 
and the flow field is asymmetric in the vertical direction. The reference velocity is the circumferential velocity component of the inner cylinder and the reference length is the gap width $d$, and the Reynolds number is defined by these reference values and the kinematic viscosity of the working fluid. The aspect ratio $\Gamma$ is the ratio of the cylinder length $L$ to the gap width $d$, and the radius ratio $\eta$ is given by $r_{\text {in }} / r_{\text {out }}$.

The flow pattern, that is the mode, is determined from the number of Taylor vortices and the rotation direction of the vortices. In the asymmetric system, when the flow near the bottom end wall is radially inward and the flow at the top is outward, the flow is a normal mode. Otherwise, the flow is an anomalous mode, and an anomalous vortex appears near the bottom end wall or the free surface, whose rotating direction is opposite to the direction of the normal mode flow. Depending on whether the anomalous vortex appears near the bottom or near the top, the flows of the anomalous mode are classified into the lower anomalous mode and the upper anomalous mode. The primary mode flow is the Taylor vortex flow that first appears when the inner cylinder is gradually accelerated from rest. This flow mode coincides with the flow mode that appears just before the appearance of the annular Couette flow when the flow at higher rotation speed is gradually decelerated [15]. Even though the geometrical parameters $\Gamma$ and $\eta$ are constant, flow modes different from the primary mode may emerge. These flow modes are called the secondary modes. The secondary mode is apt to appear when the rotation speed is increased by relatively high acceleration rates to above some critical values. Hereafter, the normal mode with $k$ vortices is denoted by $\mathrm{N} k$. The anomalous mode with $k$ vortices is represented by $\mathrm{A} k$, and the anomalous mode with a lower anomalous vortex and an upper anomalous mode are abbreviated as Alk and Auk, respectively. In the asymmetric Taylor-Couette system, the primary mode is the normal mode with odd number of vortices or the anomalous mode that includes even number of vortices and has an anomalous vortex at the top [15]. The secondary mode is the normal mode, or the anomalous mode that contains even number of vortices and has an anomalous vortex near the bottom or near the top.

\subsection{Experiment}

The experimental result shown in this paper has been obtained by one of the authors, who have been carrying out a series of experiences [15] [16]. The radius of the inner is $20 \mathrm{~mm}$ and that of the outer cylinders is $30 \mathrm{~mm}$, and the radius ratio $\eta$ is 0.667 . When the length of the cylinder is infinite, the critical Reynolds number for the onset of the axisymmetric Taylor vortex flow is 76.4. The working fluid is the glycerin solution and its kinematic viscosity is $2.19 \times 10^{-5} \mathrm{~m}^{2} / \mathrm{s}$ and the surface tension is $6.59 \times 10^{-2} \mathrm{~N} / \mathrm{m}$. The working fluid includes a small amount of aluminum flakes and the flow is visualized. Toya et al. [15] identified the primary mode and the transition processes during the deceleration of the secondary mode flows at the aspect ratio $\Gamma$ from 1.76 to 7.79 .

\subsection{Numerical Methods}

In the numerical simulation, the governing equations are the unsteady axisymmetric Navier-Stokes equations and the equation of continuity. The free surface model is the VOF (volume of fluid) and the gravitational force in the axial direction and the surface tension based on the free surface curvature are considered. Physical quantities are made dimensionless by the gap with $d$ and the maximum circumferential velocity of the inner cylinder attained during the simulation. The equations are formulated in the cylindrical system $(r, \theta, z)$ of the radial, azimuthal and axial coordinates, and the velocity components in each direction are $u, v$ and $w$. The discretization method is the finite difference method, and the time integration is the fractional step method. Initially, the velocity components are zero and the pressure is the hydrostatic pressure. The boundary condition on the solid wall is no slip condition. In order to visualize the numerical flow information, the Stokes' stream function $\varphi$ is introduced. The details of the numerical method can be found in Watanabe et al. [18].

\section{Results}

\subsection{Development of Flow at Low Aspect Ratio}

The bifurcation locus between the normal three cell mode (N3) and the normal one cell mode (N1) was numerically determined at the aspect ratio from 1.44 to 1.96 [18]. When the aspect ratio is 1.6 , the critical Reynolds number for the bifurcation lies between 830 and 840 . Figure 1 shows the contour of the stream function $\varphi$ during the time development of the flow at $\Gamma=1.6$ and $\mathrm{Re}=800$. When the dimensionless time $t$ is 0 , the inner cylinder is at rest, then it is linearly accelerated by $t=76.3$. The warm color and cold color represent the vortices 
rotating clockwise and counterclockwise, respectively. At $t=96.0$, the flow includes three vortices. The flow near the bottom end wall is inward and the flow at the top is outward, and the flow mode is N3. This flow is not stable. With time elapse, the upper two vortices cease, and a normal one cell mode appears in the final state.

The time variations of the bulk averaged energy of the axial velocity component $E_{w}$ at $\operatorname{Re}=800,810,820$, 830 and 840 are shown in Figure 2. After the rapid change at the initial stage, the energy gradually increases at least by $t=400$. The fluctuations in the profiles result from the unsteady movements of the vortical structures. When the Reynolds number is 800, the bulk energy increases during time $t$ from 600 to 700 . This time corresponds to that when the two upper vortices collapse and N1 flow appears in Figure 1. The bulk energy of the radial velocity component also showed its change. Similar increases of $E_{w}$ appear at $\operatorname{Re}=810,820$ and 830, although the onset time delays as the Reynolds number becomes large. When the Reynolds number is 840 , however, the increase of $E_{w}$ was not observed even though the simulation was continued well beyond the time range shown in Figure 2. In Figures 1 and 2, the normal one cell mode flows have larger value of the radial bulk energy than the normal three cell mode flows. This suggests that, for a fixed geometrical parameters of the aspect ratio and the radius ratio, the flow has more bulk energy of the axial velocity component as the number of the vortices becomes small. This phenomenon has also been noted by Nakase et al. [19].

\subsection{Flow at Moderate Aspect Ratio}

The development of flow at $\Gamma=5.6$ is shown in Figure 3. The inner cylinder that is at rest in the initial state is accelerated suddenly to the rotation speed corresponding to the prescribed Reynolds number 400 and 100 . When the Reynolds number is 400 in Figure 3(a), the first vortices appear near the bottom end wall and below the free surface, and eleven vortices appear by $t=50$. The vortices at the bottom and at the top rotate in the clockwise

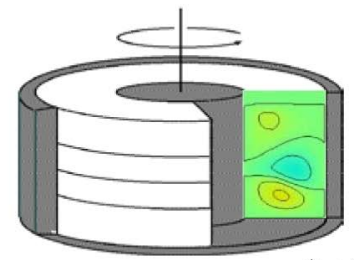

$t=64.0$

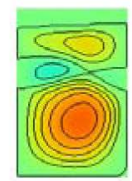

$t=688.0$

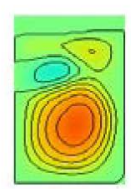

691.2

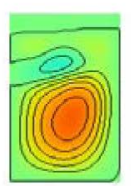

694.4

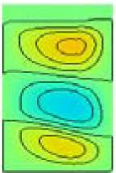

96.0

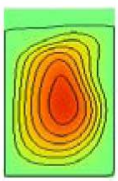

704.0

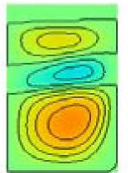

640.0

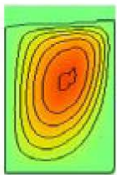

720.0

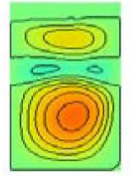

684.8

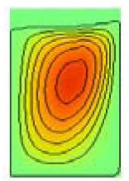

1280.0

Figure 1. Flow development at $\Gamma=1.6$ and $\mathrm{Re}=800$. The inner cylinder is linearly accelerated by $t=76.3$.

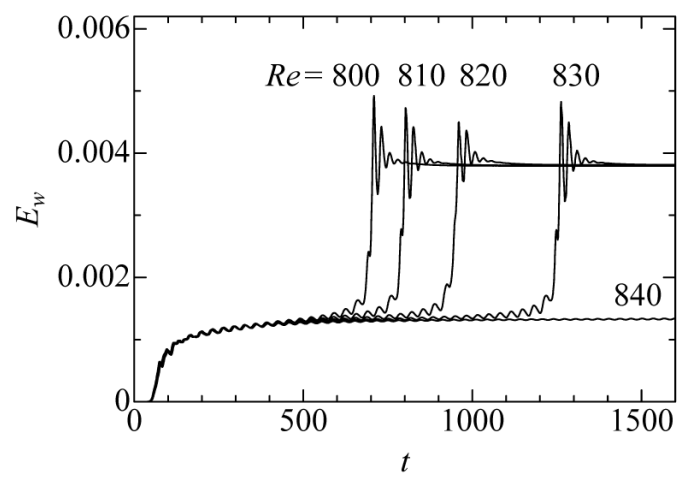

Figure 2. Time variations of bulk averaged kinetic energy of axial velocity component at $\Gamma=1.6$ and $\mathrm{Re}$ $=800,810,820,830$ and 840 . 
direction. Therefore, the flow is inward on the bottom end wall and outward at the free surface, and the flow mode is N11. The pair of the second and third vortices from the bottom and the pair of the fourth and fifth vortices have radially inward flow at the boundaries of paring vortices. These pairs of vortices are not stable, and they disappear and N7 emerges by $t=100$. This N7 flow is not stable neither. The third and fourth vortices from the free surface, which have a radially inward flow at their boundary, collapse and N5 finally appears by $t=200$. The top free surface has radially outward and its profile is higher near the outer cylinder than near the inner cylinder. During this developing process, the disappearing vortices makes a pair in which the radial flow is inward at the boundary of the vortices. Similar disappearing pair of vortices was found in the symmetric Taylor-Couette system with stationary walls at both ends of the cylinders [20].

Figure 4 shows the time variations of the bulk energy of the radial and axial velocity components of the flow at $\Gamma=5.6$ and $\operatorname{Re}=400$. The energy suddenly increase just after the start of rotation at $t=0$. From Figure 3(a), N11 appears at about $t=50$ and N9 appears at about $t$ from 100 to 140, and the profiles of the energy in Figure 4 show their maxima and minima at these time. After $t=300$, the values reach their plateaus and the flows become stable.

The development of the flow at $\mathrm{Re}=100$ is shown in Figure 3(b). The first vortex is the Ekman vortex on the stationary end wall. This vortex induces inner vortices, and the final flow includes six vortices. The top vortex rotates counterclockwise direction and this flow is Au6 that has an anomalous vortex at the top. The flow modes of N5 and Au6 were experimentally confirmed by Toya et al. [15].

When the aspect ratio is not more than 4.2, the bifurcation between the normal three cell mode and the normal one cell mode and the bifurcation between the normal five cell mode and the normal three cell mode were numerically determined [18]. In this section, the bifurcation found at $\Gamma$ from 4.6 to 6.0 is shown. Toya et al. [14] determined the critical Reynolds number for the bifurcation by gradually decreasing the Reynolds number at which the secondary mode appeared and finding the mode transition. We follow their method and numerically determine the critical Reynolds number.

The experimental result by Toya et al. shows that the primary mode at $\Gamma$ from 4.79 to 6.32 is N5. The numerical and experimental bifurcation diagrams are shown in Figures 5 and 6, respectively. In both cases, the bifurca

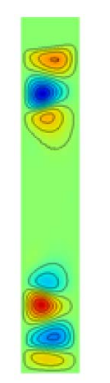

$t=30$

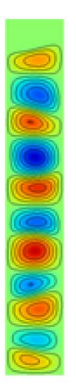

50

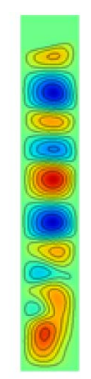

80

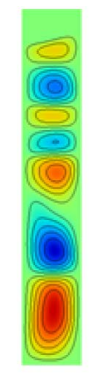

100

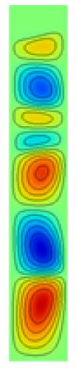

140

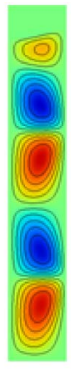

200

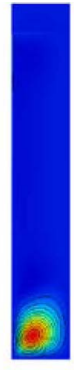

$t=10$

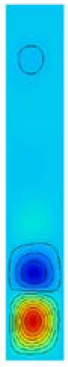

30

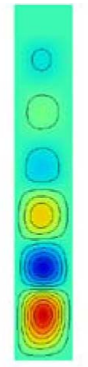

50

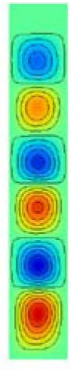

70

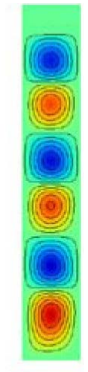

200

(a) $\mathrm{Re}=400$

Figure 3. Developments of flow at $\Gamma=5.6$. The inner cylinder is suddenly accelerated from rest.

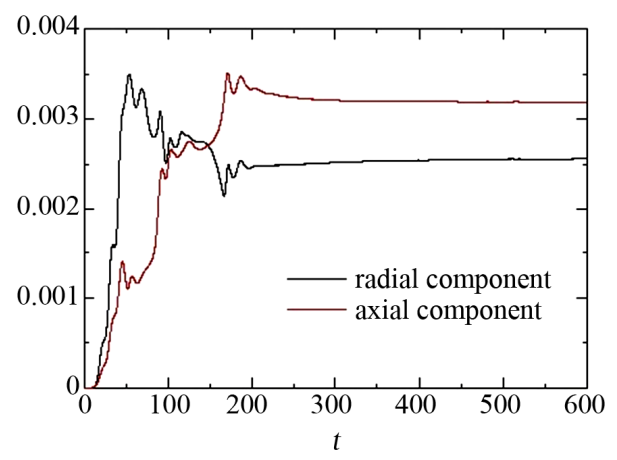

Figure 4. Time variations of bulk averaged kinetic energy of radial and axial velocity components at $\Gamma=5.6$ and $\mathrm{Re}=400$. 
tions from N7 to Au6, from Au6 to N5 and from N7 to N5 are found. The experimental and numerical results do not give good agreement about the ranges of the aspect ratio, in which the bifurcations appear, nor the critical Reynolds numbers. However, the fact that the critical Reynolds number of the transition from N7 increases as the aspect ratio decreases is well predicted. When the aspect ratio is about 6.0, the numerical and experimental result of the critical Reynolds number for the bifurcation from N7 to Au6 almost coincide with each other. While the range of the aspect ratio from 5.4 to 5.7, at which the transition from Au6 appears, is found in the numerical and experimental results, the critical Reynolds number obtained by the experiment is larger than that predicted numerically. The flow of Au6 is an anomalous flow and it is physically unstable. Therefore, it is suggested that imperfections of the experimental apparatus make the bifurcation from the unstable Au6 to the more stable N5 occur at the higher Reynolds number.

\section{Conclusions}

Numerical investigation has been carried out on the flow developing between a rotating inner cylinder and a stationary outer cylinder with vertical axes. The bottom end of the cylinders is stationary fixed wall and the top is the free surface.

Depending on the Reynolds numbers, the final flow state bifurcates into the one cell mode flow and the three cell mode flow at the aspect ratio 1.6. This shows the bifurcation mechanics of the flow even in a given geometrical configuration.

When the aspect ratio is from 5.0 to 6.0, the high Reynolds number flows developing from rest have many vortices in their transient states. It is numerically shown that, as the flow grows, a pair of vortices which have a radially inward flow between the vortices disappears and the flow state with smaller number of vortices is established. When the Reynolds number is low, the Ekman vortex emerging on the bottom stationary wall induces

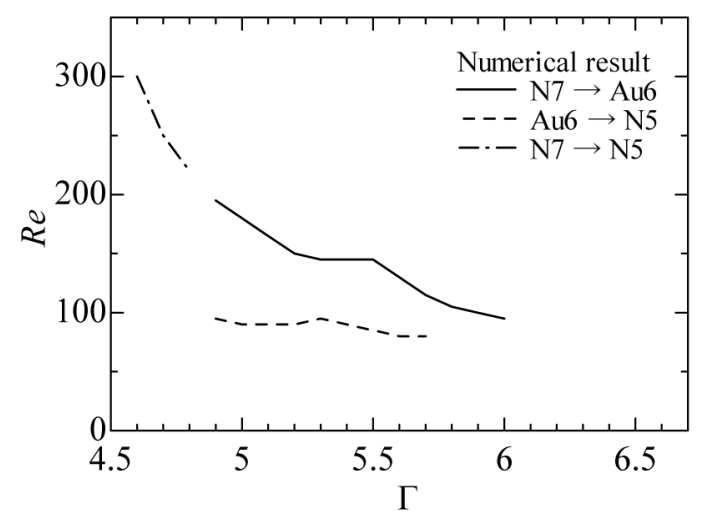

Figure 5. Numerical result of the critical Reynolds number in decelerating flows.

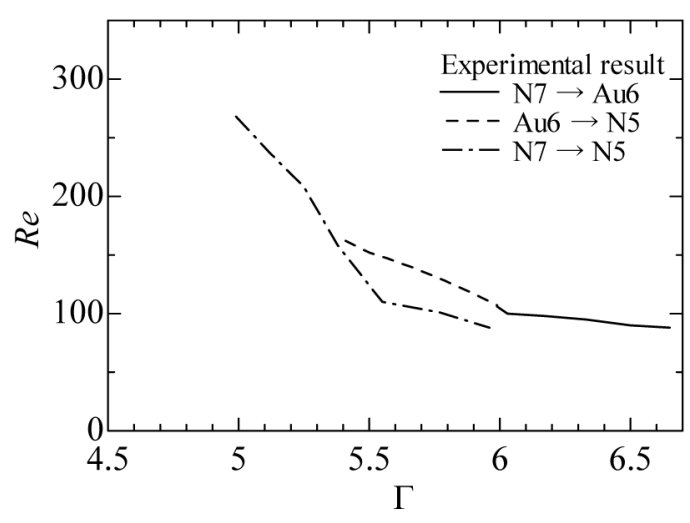

Figure 6. Experimental result of the critical Reynolds number in decelerating flows. 
interior vortices and makes the flow develop into a unique mode. The experimentally estimated critical Reynolds number for the transition from the secondary normal seven cell mode is obtained by the present numerical simulation. Especially, the transition from the normal seven cell mode to the anomalous six cell mode at the aspect ratio around 6.0 is predicted well.

\section{Funding}

This work was partly supported by JSPS KAKENHI Grant Number 23560193.

\section{References}

[1] Benjamin, T.B. (1978) Bifurcation Phenomena in Steady Flows of a Viscous Fluid: I. Theory. Proceedings of the Royal Society A, 359, 1-26. http://dx.doi.org/10.1098/rspa.1978.0028

[2] Benjamin, T.B. (1978) Bifurcation Phenomena in Steady Flows of a Viscous Fluid: II. Experiments. Proceedings of the Royal Society A, 359, 27-43. http://dx.doi.org/10.1098/rspa.1978.0030

[3] Cole, J.A. (1976) Taylor-vortex Instability and Annulus-Length Effects. Journal of Fluid Mechanics, 75, 1-15. http://dx.doi.org/10.1017/S0022112076000098

[4] Ammar, M., Ahcène, B. and Eddine, S.S. (2005) Écoulement de Taylor-Couette en Géométrie Fine et à Surface Libre. The Canadian Journal of Chemical Engineering, 83, 652-657.

[5] Deng, R., Arifin, D.Y., Mak, Y.C. and Wang, C.-H. (2009) Characterization of Taylor Vortex Flow in a Short Liquid Column. AIChE Journal, 55, 3056-3064. http://dx.doi.org/10.1002/aic.11919

[6] Caton, F., Jniaud, B. and Hopfinger, E.J. (2000) Stability and Bifurcations in Stratified Taylor-Couette Flow. Journal of Fluid Mechanics, 419, 93-124. http://dx.doi.org/10.1017/S0022112000001348

[7] Ermanyuk, E.V. and Flór, J.-B. (2005) Taylor-Couette Flow in a Two-layer Stratified Fluid: Instabilities and Mixing. Dynamics of Atmospheres and Oceans, 40, 57-69. http://dx.doi.org/10.1016/j.dynatmoce.2004.10.005

[8] Woods, A.W., Caulfield, C.P., Landel, J.R. and Kuesters, A. (2010) Non-Invasive Turbulent Mixing across a Density Interface in a Turbulent Taylor-Couette Flow. Journal of Fluid Mechanics, 663, 347-357. http://dx.doi.org/10.1017/S0022112010004295

[9] Oglethorpe, R.L.F., Caulfield, C.P. and Woods, A.W. (2013) Spontaneous Layering in Stratified Turbulent Taylor-Couette Flow. Journal of Fluid Mechanics, 721, R3-1-12.

[10] Djéridi, H., Favé, J.-F., Billard, J.-Y. and Fruman, D.H. (1999) Bubble Capture and Migrationin Couette-Taylor Flow. Experiments in Fluids, 26, 233-239. http://dx.doi.org/10.1007/s003480050284

[11] Djéridi, H., Gabillet, C. and Billard, J.Y. (2004) Two-phase Couette-Taylor Flow: Arrangement of the Dispersed Phase and Effects on the Flow Structures. Physics of Fluids, 16, 128-139. http://dx.doi.org/10.1063/1.1630323

[12] Atkhen, K., Fontaine, J. and Wesfreid, J.E. (2000) Highly Turbulent Couette-Taylor Bubbly Flow Patterns, Journal of Fluid Mechanics, 442, 55-68. http://dx.doi.org/10.1017/S0022112000001592

[13] Sugiyama, K., Calzavarini, E. and Lohse, D. (2008) Microbubbly Drag Reduction in Taylor-Couette Flow in the Wavy Vortex Regime. Journal of Fluid Mechanics, 608, 21-41. http://dx.doi.org/10.1017/S0022112008001183

[14] Nakamura, I., Toya, Y., Yamashita, S. and Ueki, Y. (1990) An Experiment on a Taylor Vortex Flow in a Gap with a Small Aspect Ratio (Bifurcation of Flows in a Symmetric System). JSME International Journal. Series II, 33, 685-691.

[15] Nakamura, I., Toya, Y., Yamashita, S. and Ueki, S. (1989) An experiment on a Taylor Vortex Flow in a Gap with a Small Aspect Ratio (Instability of Taylor Vortex Flows). JSME International Journal. Series II, 23, 388-394.

[16] Toya, Y., Nakamura, I., Yamashita, S. and Ueki, S. (1994) An Experiment on a Taylor Vortex Flow in a Gap with a Small Aspect Ratio: Bifuration of Flows in an Asymmetric system. Acta Mechanica, 102, 137-148. http://dx.doi.org/10.1007/BF01178523

[17] Linek, M. and Ahlers, G. (1998) Boundary Limitation of Wave Numbers in Taylor-vortex Flow. Physical Review E, 58, 3168-3174. http://dx.doi.org/10.1103/PhysRevE.58.3168

[18] Watanabe, T. and Toya, Y. (2012) Vertical Taylor-Couette Flow with Free Surface at Small Aspect Ratio. Acta Mechanica, 222, 347-353. http://dx.doi.org/10.1007/s00707-011-0569-9

[19] Nakase, M., Makabe, R. and Takeshita, K. (2013) Relation between Oil-Water Flow and Extraction Performance in Liquid-Liquid Countercurrrent Centrifugal Extractor with Taylor Vortices. Journal of Nuclear Science and Technology, 50, 287-295. http://dx.doi.org/10.1080/00223131.2013.772445

[20] Watanabe, T., Furukawa, H. and Nakamura, I. (2002) Nonlinear Development of Flow Patterns in an Annulus with Decelerating Inner Cylinder. Physics of Fluids, 14, 333-341. http://dx.doi.org/10.1063/1.1416498 